\title{
Optimization of Near-Field Scanning Optical Lithography
}

\author{
Ben S. Routley ${ }^{a, b}$, John L. Holdsworth ${ }^{b}$ and Andrew J. Fleming ${ }^{a}$ \\ ${ }^{a}$ School of Electrical Engineering and Computer Science, University of Newcastle, Callaghan, \\ Australia; \\ ${ }^{b}$ School of Mathematical and Physical Sciences, University of Newcastle, Callaghan, Australia
}

\begin{abstract}
This article describes two- and three-dimensional optical simulations for determining optimal conditions for near-field scanning optical lithography. It was found that a combination of 30-nm thick photoresist and 50-nm thick anti-reflective coating will yield optimal results with $405 \mathrm{~nm}$ incident light and a hollow-cantilever probe with 100-nm aperture width. In addition to identifying the optimal conditions, the sensitivity of the resolution with respect to each parameter is explored and plotted. The mechanisms behind each trend are described with supporting simulation data.
\end{abstract}

Keywords: Near-field scanning optical lithography, Simulation

\section{INTRODUCTION}

Near-field scanning optical lithography (NSOL) offers a pathway to integrated circuit (IC) prototyping with $30 \mathrm{~nm}$ feature sizes. ${ }^{1}$ NSOL is an extension of near-field scanning optical microscopy which uses nano-structured probes to confine light below the diffraction limit which is commonly expressed as $\lambda / 2$ where $\lambda$ is the wavelength of the incident light. Typically this is achieved with an aperture width of less than the diffraction. At close proximity to this aperture, the transmitted light is a non travelling wave or a evanescent field. At far proximity to the aperture this evanescent field will scatter and diffract out into a travelling wave and hence it is key that the aperture be kept in close proximity to the sample. A common technique used to maintain this close proximity is to use an atomic force microscope (AFM). ${ }^{2}$ Due to the use of AFM feedback systems, many NSOM probes take the form of modified AFM probes. In Figure 1, two NSOM probe designs based on AFM probes are illustrated.

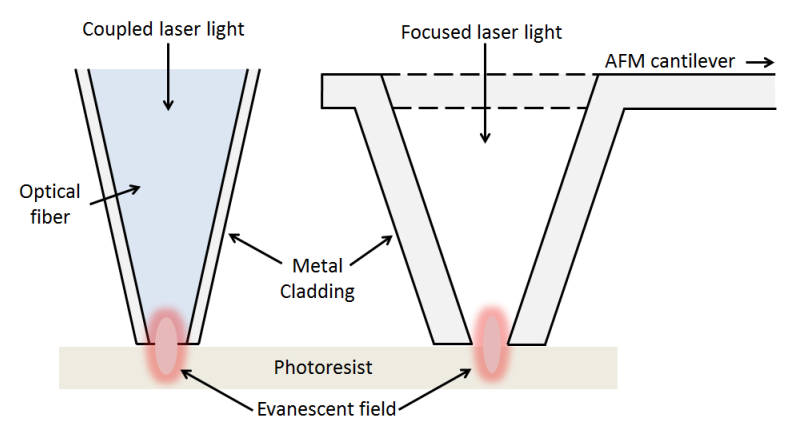

Figure 1. Different NSOL configurations using: left, tapered optical fibre probe; right, hollow-cantilever probe.

Further author information: (Send correspondence to B.S.R.)

B.S.R.: E-mail: Ben.Routley@newcastle.edu.au 
Although current optical techniques offer 30nm resolution with much higher throughput they require a unique mask for each IC design. ${ }^{3}$ Prototype mask sets are typically costly and require significant lead times which reduces the suitability in early stage prototyping. Since NSOL is a direct write technique, no mask is required. Other direct write techniques include electron-beam lithography, x-ray lithography and ion-beam lithography. These techniques require a high vacuum which involves a high capital cost. Advantageously, NSOL can be performed at atmospheric pressure and is readily compatible with established mask-based manufacturing techniques. Traditionally, NSOL is considered to be a serial process with a relativity low throughput. However NSOL can be performed in parallel with the use of an array of near-field probes. ${ }^{4}$ Advances in AFM bandwidth ${ }^{5,6}$ and the speed of nanopositioning systems ${ }^{7}$ may allow thousands or millions of points to be exposed per second.

As NSOL is an extension of NSOM it suffers from the same issues. One such issue is that the amount of light transmitted through the aperture is dependent on the distance between the sample and also the aperture; and also the optical properties of the sample. ${ }^{8,9}$ It is for these reasons that the sample be flat and homogeneous. The resolution of NSOM is dependent on many factors but resolution below the aperture width is achievable. ${ }^{10}$ Resolutions below the aperture width have also been achieved for NSOL. ${ }^{1}$ In order to achieve the best resolution, many system properties require optimization. The key properties are the photoresist layer thickness, antireflective coating thickness, aperture width and wavelength of the incident light. Optimizing all of these properties would be a difficult task if done solely experimentally; therefore, this work seeks to develop a simulation model.

A simulation model based on the finite-difference frequency-domain (FDTD) method has been previously used to explain the presence of artefacts in NSOM results. ${ }^{9}$ FDTD has also been used to predict the point spread function for a NSOL system that used a bowtie optical nano-antenna. ${ }^{11}$ FDTD solves Maxwell's equations for a discrete grid of material properties by stepping the equations through time. This allows for the calculation of the electric and magnetic fields at each point on the grid and thus solutions for complex geometries can be found. Although FDTD has been proven to work it does have long computational times for systems that include metals. These long computational times are due to the high number of iterations required to obtain the steady state solution. The time step size is dependent on the smallest refractive index and thus when a metal which has refractive index of less than one is added the computational time increases significantly. A suitable alternative is to use a frequency domain solver such as the finite-domain frequency-domain (FDFD) method which directly produces the steady state solution. However, FDFD still suffers when metals are added to the system as the grid size needs to be small enough to sample the waves propagating in the metal, resulting in large memory requirements and long computational times. However, if the grid size is non-uniform this wave sampling issue can be overcome.

\section{MODELLING APPROACH}

As discussed in the introduction, a frequency-domain method that has a non-uniform grid is ideal and thus a model was developed within the COMSOL multiphysics framework. COMSOL has a frequency-domain solver suitable for optics built in and uses an adaptive mesh to represent the geometry, allowing for high resolution only where it is needed which greatly reduces the memory requirements and computation time. COMSOL solves Maxwell's equations in the frequency domain and produces the steady state solutions for the electric field at each element of the mesh thus producing a map of the electric field. By multiplying the modulus of this map squared with the corresponding imagery dielectric constant, a map that is proportional to the absorption at each grid point is produced. ${ }^{12}$ An example of an absorption map is Figure 8 (1) and Figure 8 (2). These maps can then be post-processed to produce the absorption profiles shown in Figure 3 (left). These profiles can be used to calculate the full width half maximum (FWHM). Although the FWHM is not equal to the resulting feature size, it is a useful metric for comparing arrangements. The FWHM was calculated at the photoresist/anti-reflective coating interface as this will be the maximum.

In order to solve Maxwell's equations the complex refractive index for the materials is required. The photoresist under consideration is AZ-701 from Microchemicals GmbH. The bleached state properties were chosen so that 
the model represented the worse case of scattering. The anti-reflective coating used was AZ barli II which is also supplied by Microchemicals GmbH. The NSOM probe was modelled to represent a NT-NDT hollow cantilever probe (HPC) which can be seen in Figure 1 right). It was assumed that this probe consisted of aluminium in the bulk form, thus ignoring surface oxides. The optical properties for the photoresist and the anti-reflective coating were taken from the manufactures website. The optical properties for aluminium were taken from. ${ }^{13}$ The optical properties for the silicon substrate were taken from. ${ }^{14}$

The parameters that were explored included the wavelength of the incident light, the thickness of the photoresist, the thickness of the anti-reflective coating, the separation between the probe and photoresist, and the aperture width. The optimization process was done in this order by taking the best result and using it on the proceeding simulations. The aperture width was considered last as it is physically fixed to $100 \mathrm{~nm}$ by the manufacturer. These simulations were preformed under 2-dimensional conditions to increase the computational performance and allow for high density meshes. The 2-dimensional results were then validated by comparing them to 3-dimensional results for two different cases. The geometry for the 2-dimensional case was generated within COMSOL which allows for parameters to be easily adjusted. However, due to the complex geometry required for the probe in the 3-dimensional simulations, Autodesk Inventor was used to model the probe which was then imported into COMSOL. The imported probe and critical dimensions are shown in Figure 2.
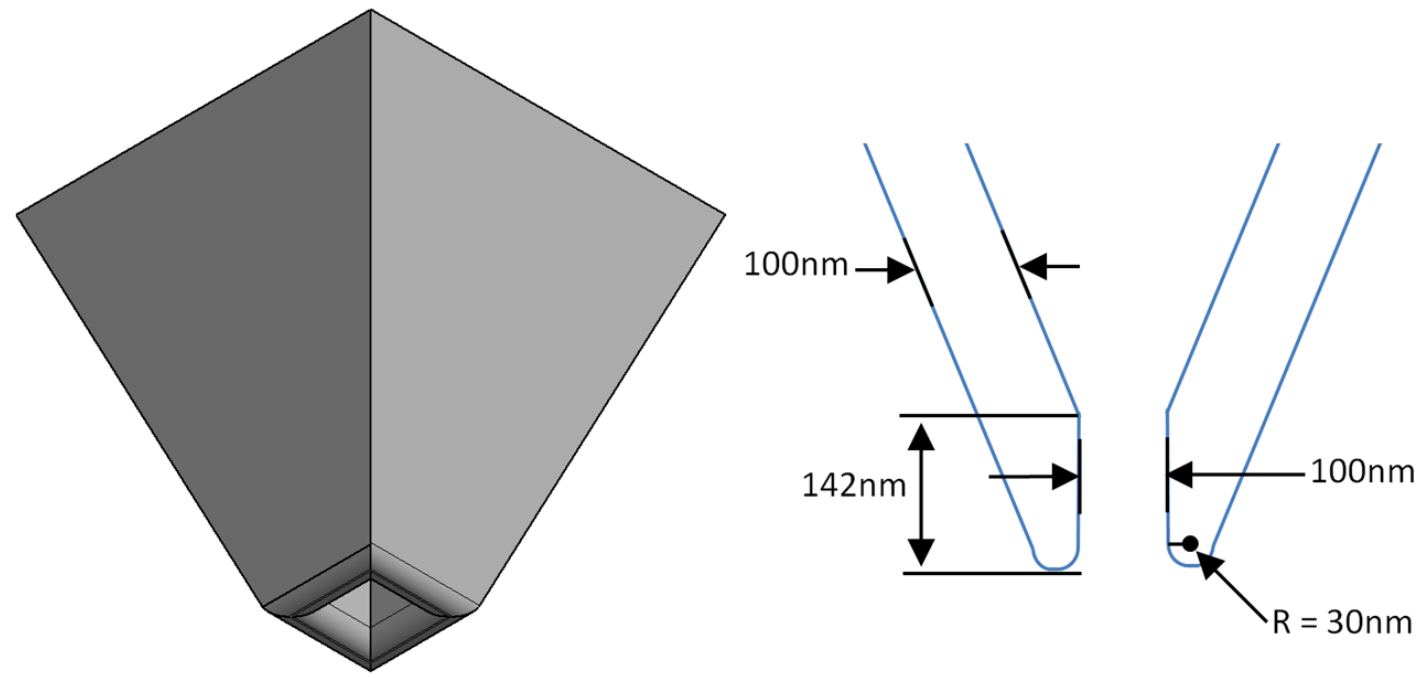

Figure 2. Left, a 3-dimensional representation of the probe geometry used for the 3-dimensional case. Right, a diagram highlighting the critical dimensions used for both the 2-dimensional and 3-dimensional cases.

\section{RESULTS}

\subsubsection{2-DIMENSIONAL}

The effect of the incident light's wavelength was first explored. Three wavelengths were tested: $365 \mathrm{~nm}, 405 \mathrm{~nm}$ and $436 \mathrm{~nm}$. These wavelengths were chosen as they are commonly used within the lithography industry. The results shown in Figure 3 (left) indicate that the wavelength has very little effect on the optical confinement achieved by the probe, therefore readily available $405 \mathrm{~nm}$ laser diodes can be utilized and thus $405 \mathrm{~nm}$ light was used for the remaining simulations. Next, the effect of photoresist thickness was tested using an anti-reflective coating thickness of $200 \mathrm{~nm}$ and probe separation of $5 \mathrm{~nm}$. As indicated in Figure 3 (right) the photoresist thickness drastically effects the FWHM. This effect is due to fact that the evanescent field produced by the probe will scatter away form the probe and begin to undergo diffraction, hence shorter optical paths result in smaller FWHM. However, it appears that if the photoresist is made $15 \mathrm{~nm}$ thick the FWHM appears to increase and hence a $30-\mathrm{nm}$ thick photoresist was chosen for the remaining simulations. 
Using a wavelength of $405 \mathrm{~nm}$, a photoresist thickness $30 \mathrm{~nm}$, and a probe separation of $5 \mathrm{~nm}$, the effect of anti-reflective coating thickness was tested. The results in Figure 4 (left) indicate that there exists a complex relationship between the FWHM and the anti-reflective coating thickness. There is a FWHM maxima for antireflective coating thicknesses of $120 \mathrm{~nm}$ and $20 \mathrm{~nm}$. A minima for a thickness exists at $50 \mathrm{~nm}$. The first maxima occurs at around $120 \mathrm{~nm}$, which is approximately half a wavelength inside the anti-reflective coating and thus is most likely a result of an interference lobe forming inside the anti-reflective coating. This lobe can be seen in Figure 5 (3). The minima that occurs for a thickness of approximately $50 \mathrm{~nm}$ is likely a result of this interference lobe not forming as it can not exist inside this layer. As a result, the reaming light is scattered directly back up the probe or absorbed at the edge of the anti-reflective coating. This removal of the lobe can be see in Figure 5 (2). The maxima that occurs for thicknesses less than $10 \mathrm{~nm}$ is likely a result of leaking or coupled light from the aluminium coating of the probe scattering off the silicon as there is too little anti-reflective coating to absorb it. This idea is confirmed by the results shown in Figure 8 as there are two peaks formed in the absorption profile. These peaks correspond to the edges of the probe indicating that light is being leaked or coupled through the aluminium. From these results the anti-reflective coating was chosen to be 50-nm thick in the remaining simulations.
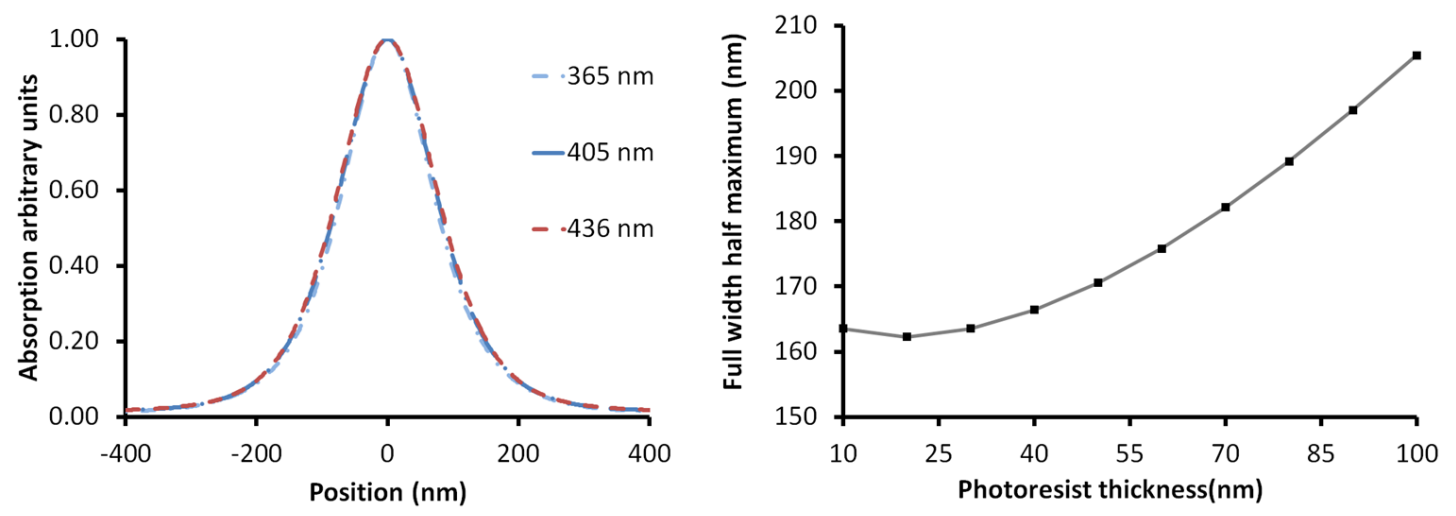

Figure 3. Left, absorption profiles at the boundary between the photoresist layer and the anti-reflective layer versus laser wavelength. Right, full width half maximum at the boundary between the photoresist layer and the anti-reflective layer versus photoresist layer thickness.
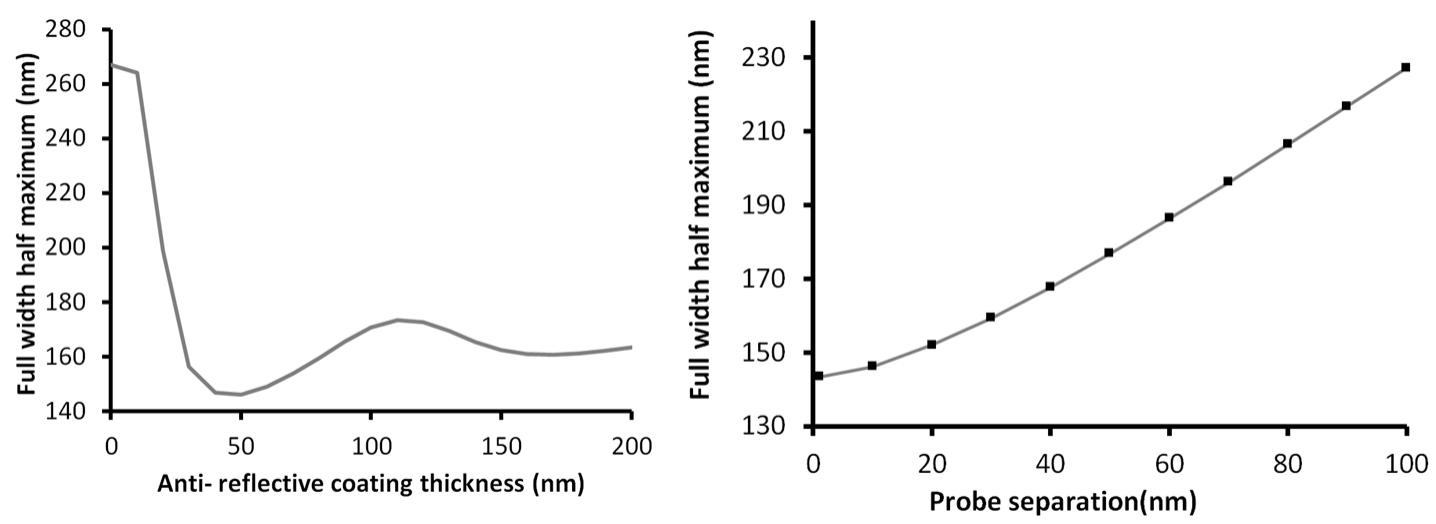

Figure 4. Left, full width half maximum at the boundary between the photoresist layer and the anti-reflective layer versus anti-reflective layer thickness. Right, full width half maximum at the boundary between the photoresist layer and the anti-reflective layer versus separation distance between the sample and the probe. 
1)

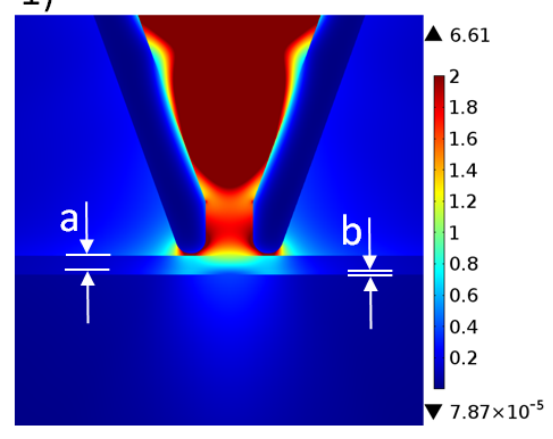

2)

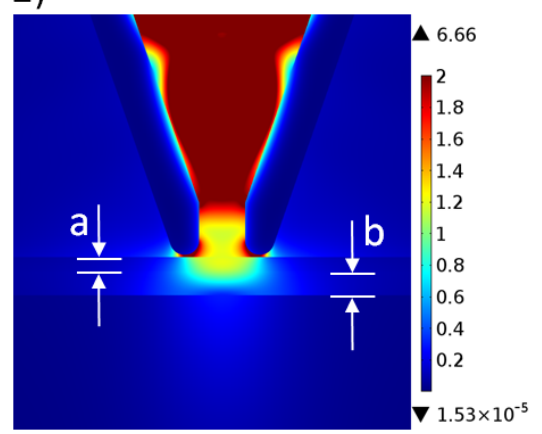

3)

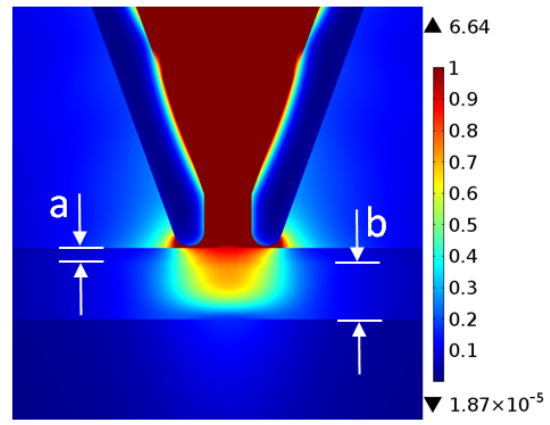

Figure 5. 1) electric field map for a anti-reflective coating with a thickness of $10 \mathrm{~nm}$. 2) electric field map for a antireflective coating with a thickness of $50 \mathrm{~nm}$. 3) electric field map for a anti-reflective coating with a thickness of $120 \mathrm{~nm}$. For all maps region (a) represents a photoresist layer with a thickness of 30nm and region (b) represents the anti-reflective coating.

Next, the effect of probe separation was explored using a wavelength of $405 \mathrm{~nm}$, a photoresist thickness of $30 \mathrm{~nm}$, and anti-reflective coating thickness of $50 \mathrm{~nm}$. The results shown in Figure 4 (left) indicate that by increasing the probe separation, the FWHM is increased. This is because the evanescent field can scatter out and diffract more readily when there is a longer optical path length. This means that an AFM system using contact mode is desirable over a tapping mode system. Up to this point, all simulations were performed using a probe aperture width of $100 \mathrm{~nm}$ as this is fixed by the manufacturer. However, different aperture sizes were examined using a wavelength of $405 \mathrm{~nm}$, a photoresist thickness of $30 \mathrm{~nm}$, an anti-reflective coating thickness of $50 \mathrm{~nm}$, and a probe separation of $5 \mathrm{~nm}$. The results in Figure 6 indicate that the FWHM can be decreased by reducing the aperture. However, it should be noted that the intensity of the transmitted light is inversely proportional to the sixth power of the aperture width so a reduction will have significant effects on throughput.

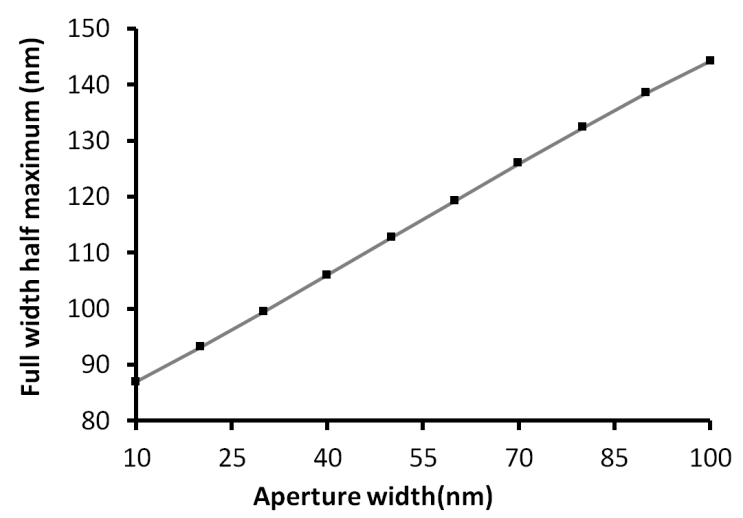

Figure 6. Full width half maximum at the boundary between the photoresist layer and the anti-reflective layer versus probe aperture width. 


\subsubsection{3-DIMENSIONAL}

Two different cases were chosen to validate the 2-dimensional results with a 3 -dimensional model. Both cases used a wavelength of $405 \mathrm{~nm}$, a photoresist thickness of $30 \mathrm{~nm}$, a probe separation of $5 \mathrm{~nm}$, and an aperture width of $100 \mathrm{~nm}$. The difference between the two cases was the presence of an anti-reflective coating. One case considered a $200 \mathrm{~nm}$ thick anti-reflective coating whilst the other used no anti-reflective coating. These cases were chosen as they gave very different results for the 2-dimensional simulations. The 3 -dimensional simulation is represented Figure 7. It can be observed that in region (c) the grid size was relatively large in order to fit the simulation in the computer memory. The overall physical size of the simulation was also drastically decreased to save memory. Even with all of these compromises, the 3-dimensional model still required approximately 5 times more memory than the 2-dimensional model, it also took 20 times longer to run. This increase in computational time makes the 2-dimensional model more desirable for optimising parameters.
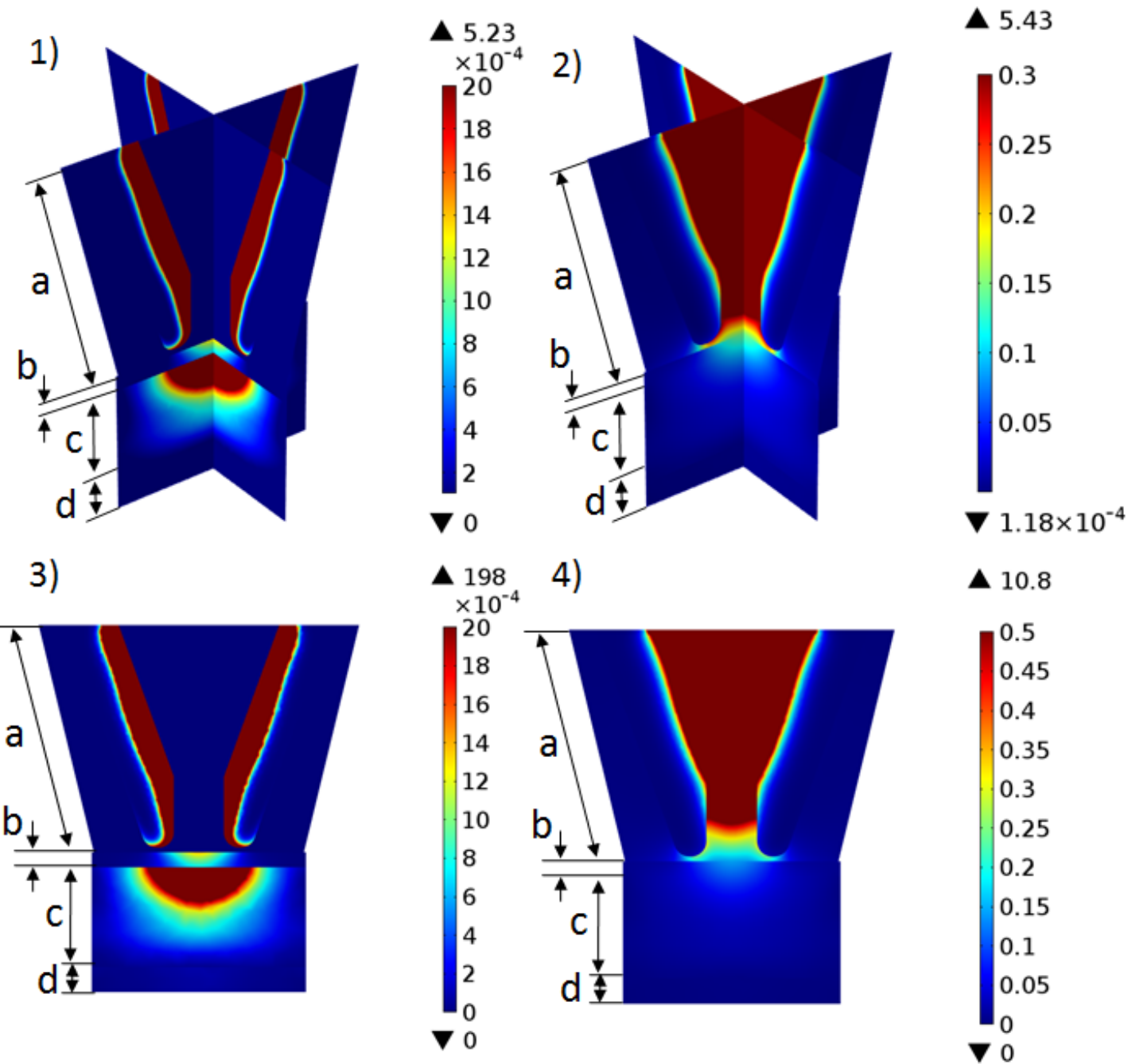

Figure 7. 1) 3D absorption map for $\mathrm{X}$ and $\mathrm{Y}$ planes, 2) 3D electric field map for $\mathrm{X}$ and $\mathrm{Y}$ planes, 3) 3D absorption map for the XY plane, 4) 3D electric field map for the XY plane. For all maps a) $500 \mathrm{~nm}$ layer of air and aluminium; b) $30 \mathrm{~nm}$ layer of photoresist; c) $200 \mathrm{~nm}$ layer of anti-reflective coating; d) $50 \mathrm{~nm}$ layer of silicon. 
In order to compare the two- and three-dimensional simulations, the absorption profiles at the surface of the photoresist layer were examined. For the 3-dimensional model, an absorption map was obtained from a slice along the x-axis. This slice is plotted in Figure 7. The resulting profiles are plotted in Figure 8. These results indicate that for both cases, the 2-dimensional results follow the same trends as the 3-dimensional results. The fact that these trends are common to both models indicates that the 2-dimensional model is suitable for optimising parameters. The discrepancies between the two models may be caused by a number of issues. For example, when the 2-dimensional model is represented in a 3-dimensional space, it is represented by a infinite channel as opposed to an aperture. Given that the electric field is solved for a 3-dimensional space, this is likely to cause discrepancies. A second possibility is due to the reduction in mesh density which is required to fit the simulation inside the computer memory, which may have resulted in simulation artefacts even though the simulations converged.

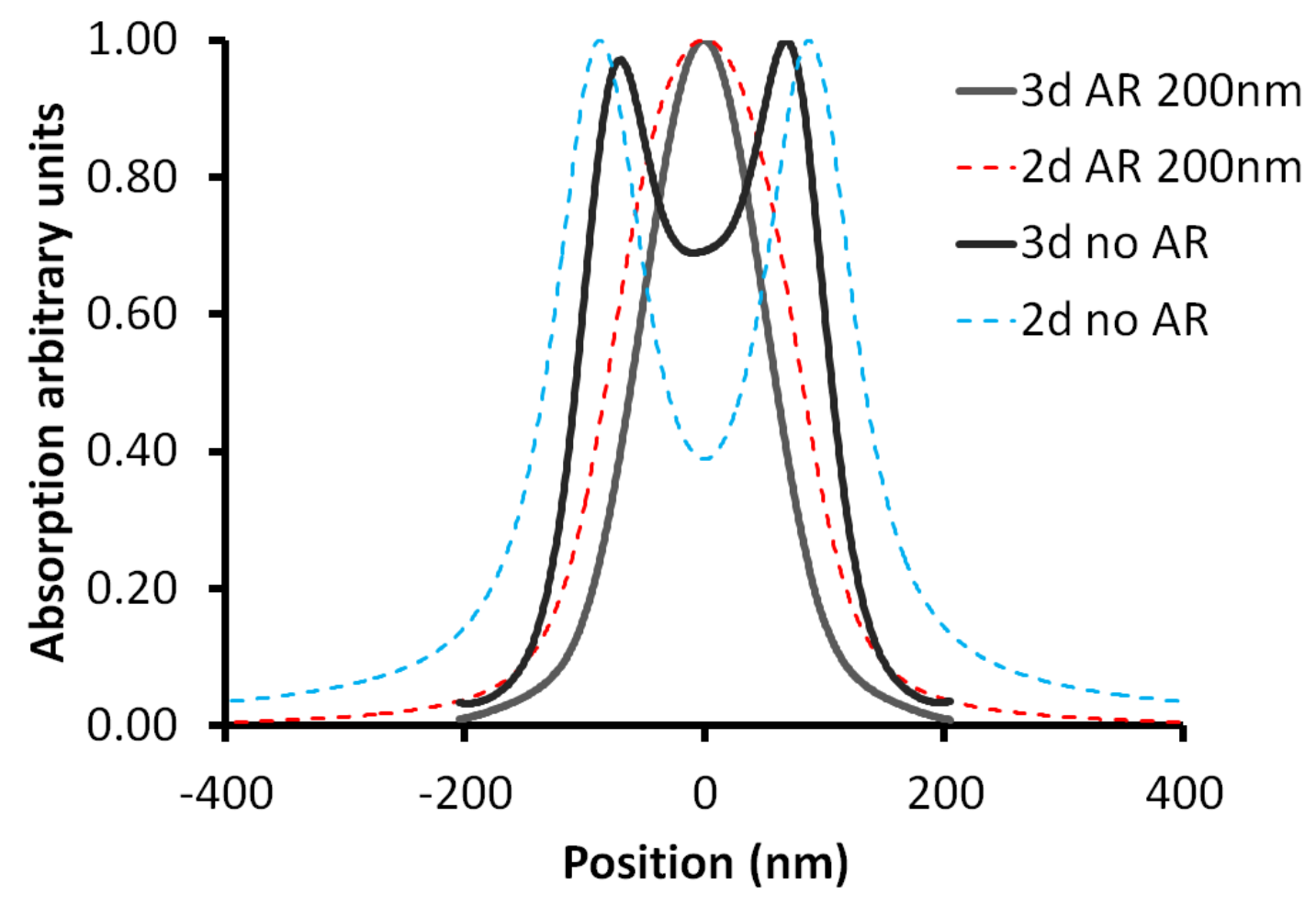

Figure 8. A comparison between the absorption profile at the boundary between the photoresist layer and the antireflective/silicon layer for the 3 -dimensional case and the 2-dimensional case.

\section{CONCLUSION}

Using the COMSOL multiphysics framework, a two- and a three-dimensional optical model was created for a near field optical lithography system. It was found that the two-dimensional model was an appropriate approximation to the three-dimensional model for the purpose of optimizing parameters. These parameters included the incident light wavelength, photoresist thickness, the anti-reflective coating thickness, probe separation distance, and probe aperture width. It was found that an optimal practical resolution should occur with a incident wavelength of $405 \mathrm{~nm}$, a photoresist thickness of $30 \mathrm{~nm}$, an anti-reflective coating thickness of $50 \mathrm{~nm}$, a probe separation distance of $5 \mathrm{~nm}$ and, and a probe aperture width of $100 \mathrm{~nm}$.

In future work, the model predictions will be compared to experimental lithographic results with a scanning near field optical microscope. 


\section{REFERENCES}

[1] Lin, Y., Hong, M., Wang, W., Law, Y., and Chong, T., "Sub-30?nm lithography with near-field scanning optical microscope combined with femtosecond laser," Applied Physics A 80, 461-465 (Feb. 2005).

[2] Lewis, A., Taha, H., Strinkovski, A., Manevitch, A., Khatchatouriants, A., Dekhter, R., and Ammann, E., "Near-field optics: from subwavelength illumination to nanometric shadowing," Nature Biotechnology 21, 1378-1386 (Nov. 2003).

[3] Owa, S., Wakamoto, S., Murayama, M., Yaegashi, H., and Oyama, K., "Immersion lithography extension to sub-10nm nodes with multiple patterning," 9052, 90520O-90520O-9 (2014).

[4] Huo, F., Zheng, G., Liao, X., Giam, L. R., Chai, J., Chen, X., Shim, W., and Mirkin, C. A., "Beam pen lithography," Nature Nanotechnology 5, 637-640 (Sept. 2010).

[5] Fleming, A. J., Kenton, B. J., and Leang, K. K., "Bridging the gap between conventional and video-speed scanning probe microscopes," Ultramicroscopy 110, 1205-1214 (August 2010).

[6] Kenton, B. J., Fleming, A. J., and Leang, K. K., "A compact ultra-fast vertical nanopositioner for improving SPM scan speed," Review of Scientific Instruments 82(12), 123703(1-8) (2011).

[7] Fleming, A. J. and Leang, K. K., [Design, Modeling and Control of Nanopositioning Systems], Springer, London, UK (2014).

[8] Hecht, B., Bielefeldt, H., Inouye, Y., Pohl, D. W., and Novotny, L., "Facts and artifacts in near-field optical microscopy," Journal of Applied Physics 81(6), 2492-2498 (1997).

[9] Klapetek, P. and Bur??k, J., "Near-field scanning optical microscopy studies of thin film surfaces and interfaces," Applied Surface Science 254, 3681-3684 (Apr. 2008).

[10] Berriman, G., Routley, B., Clothier, S., Holdsworth, J. L., Belcher, W. J., Zhou, X. J., and Dastoor, P. C., "Development of a multi-wavelength photocurrent mapping system," Measurement Science and Technology 24, 105604 (Oct. 2013).

[11] Sundaramurthy, A., Schuck, P. J., Conley, N. R., Fromm, D. P., Kino, G. S., and Moerner, W. E., "Toward nanometer-scale optical photolithography: Utilizing the near-field of bowtie optical nanoantennas," Nano Letters 6, 355-360 (Mar. 2006).

[12] Ferry, V. E., Sweatlock, L. A., Pacifici, D., and Atwater, H. A., "Plasmonic nanostructure design for efficient light coupling into solar cells," Nano Letters 8, 4391-4397 (Dec. 2008).

[13] Rakic, A. D., Djurisis, A. B., Elazar, J. M., and Majewski, M. L., "Optical properties of metallic films for vertical-cavity optoelectronic devices," Applied Optics 37, 5271-5283 (Aug. 1998).

[14] Vuye, G., Fisson, S., Nguyen Van, V., Wang, Y., Rivory, J., and Abel?s, F., "Temperature dependence of the dielectric function of silicon using in situ spectroscopic ellipsometry," Thin Solid Films 233, 166-170 (Oct. 1993). 